\title{
Búsqueda, evaluación y selección de información digital en un aula de secundaria: Tensiones entre la práctica y el discurso del profesorado y el alumnado
}

\author{
Maite López Flamarique \\ maite.lopez@unavarra.es \\ Universidad Pública de Navarra, España \\ Txema Egaña \\ tegana@mondragon.edu \\ Mondragon Unibertsitatea, España \\ Eneritz Garro Larrañaga \\ egarro@mondragon.edu \\ Mondragon Unibertsitatea, España
}

\begin{abstract}
Resumen
En un contexto comunicativo donde cada vez hay más información disponible, uno de los objetivos de la escuela es que el alumnado desarrolle una actitud crítica. Los estudios sobre alfabetización informacional en las aulas han observado inconsistencias entre el discurso y la práctica didáctica, y entre el uso previsto y el real de las TIC. Este estudio busca contrastar el discurso y la práctica del alumnado y del profesorado al buscar, evaluar y seleccionar información digital en un aula de secundaria. Se ha utilizado una triangulación de datos multifuente (grupos de discusión, registro de clases, autoconfontación). Los resultados señalan que el alumnado muestra interés por la tarea, si bien su percepción se ve condiciona por los adultos. Los estudiantes tienen dificultades en la interpretación de vídeos y gráficos y le dan poco valor a la autoría del texto. Por otro lado, los docentes ven cuestionado su rol y aunque son conscientes de las carencias del alumnado se muestran reticentes a trabajarlas en el aula. Las conclusiones apuntan que es necesaria una didáctica de la alfabetización informacional que parta de las prácticas reales e intereses del alumnado. Para ello, se requiere un cambio de rol y de creencias del profesorado.
\end{abstract}

\section{Palabras clave}

Búsqueda de información; evaluación de información; TIC; profesorado; estudiantes. 


\title{
Search, evaluation and selection of digital information in a secondary classroom: Tensions between the practice and the discourse of teachers and students
}

\author{
Maite López Flamarique \\ maite.lopez@unavarra.es \\ Universidad Pública de Navarra, España \\ Txema Egaña \\ tegana@mondragon.edu \\ Mondragon Unibertsitatea, España \\ Eneritz Garro Larrañaga \\ egarro@mondragon.edu \\ Mondragon Unibertsitatea, España
}

\begin{abstract}
In a communicative context where more and more information is available, one of the objectives of the school is for the students to develop a critical attitude. Studies on information literacy in classrooms have observed inconsistencies between discourse and didactic practice, and a significant distance between the intended and actual use of ICT in class. Therefore, this study seeks to contrast the discourse and practice of both students and teachers when searching, evaluating and selecting digital information in a secondary classroom. This is a case study that has used a multi-source data triangulation (discussion groups, activity record, self-confrontation). The results indicate that the students show interest in the task, although their perception is conditioned by the adults. Students have difficulties in interpreting videos and graphics and give little value to the authorship of the text. On the other hand, teachers have questioned their role and although they are aware of the lacks of students, teachers are reluctant to work in the classroom. The conclusions suggest that it is necessary to develop a didactic of informational literacy based on the real practices and interests of the students. For this, a change of role and beliefs of the teaching staff is required.
\end{abstract}

\section{Keywords}

Information search; information evaluation; ICT; teachers; students. 
Búsqueda, evaluación y selección de información digital en un aula de secundaria: Tensiones entre la práctica y el discurso del

\section{Introducción}

Vivimos en una sociedad donde cada vez hay más información disponible y en la que la proliferación de noticias falsas o prácticas comunicativas de posverdad (Montoya Camacho, 2017) hacen que la búsqueda, evaluación y selección de información sea una competencia esencial para el desarrollo de una ciudadanía crítica, participativa y reflexiva. En este contexto, los jóvenes que hoy en día están en las aulas de Educación Secundaria han crecido rodeados de pantallas que utilizan en su día a día (Gairín \& Mecader, 2018). Perciben que usar internet en su proceso de aprendizaje hace que aprender sea más interesante, pero no necesariamente más entretenido, y creen que aprender a utilizar bien internet les ayudará a ser mejores profesionales en el futuro (Gialamas, Nikolopoulou, \& Koutromanos, 2013). Sin embargo, Campos Martínez (2015) ha observado también en los estudiantes un discurso negativo sobre el uso de las TIC en la escuela, que parece influenciado por el pensamiento de los adultos.

En cuanto a su práctica, la mayoría de los jóvenes confía en sus habilidades para la búsqueda de información en internet, si bien solo el $2 \%$ utiliza habilidades de pensamiento crítico durante la búsqueda de información digital. De aquí se infiere que existe una brecha entre la utilización de las TIC y su uso crítico (Watkins, Engel, \& Hastedt, 2015). Es más, en ocasiones, los estudiantes perciben que son más competentes que el profesorado (Fraga Varela \& Gewerc Barujel, 2013). Sin embargo, al analizar el discurso de los estudiantes, «se desprende que la idea de competencia digital que poseen es una visión reducida de la misma» (Fraga-Varela \& Rodríguez-Groba, 2017, p. 135). En este sentido, Kirschner y Bruyckere (2017) consideran que los «nativos digitales» no existen: «though learners in this generation have only experienced a digital connected world, they are not capable of dealing with modern technologies in the way which is often ascribed to them» (Kirschner \& Bruyckere, 2017, p. 140).

En el contexto escolar, el alumnado, en su actividad escolar, con frecuencia se enfrenta al reto de buscar, seleccionar y utilizar información en internet (Pow \& Li, 2015)(Pow \& Li, 2015; Pow, Li, \& Fung, 2009), tarea difícil especialmente para estudiantes de educación media (Eagleton, Guinee, \& Langlais, 2003). En general, los estudiantes tienen pocas destrezas para definir y seleccionar información confiable (Rodrigues, 2014) y encuentran difícil diferenciar entre opiniones y datos (Kim \& Sin, 2011). La utilización de información proveniente de fuentes diversas y la comparación de contenidos les resulta particularmente exigente (Hatlevik \& Hatlevik, 2018) y suelen priorizar la selección de información fácil de usar antes que la de calidad (Biddix, Chung, \& Park, 2011). Valverde Crespo y González Sánchez (2016) concluyen que el alumnado utiliza Wikipedia como una de sus primeras fuentes por su accesibilidad y porque encuentran información sobre cualquier tema; el alumnado manifiesta no tener problemas para entender el lenguaje y contenido de la enciclopedia virtual.

Parece evidente que los discentes requieren del apoyo y andamiaje del profesorado para aprender a manejar la información de forma eficaz y crítica (Hatlevik \& Hatlevik, 2018). Como apuntan Pow y Li (2015, p. 453), el profesorado debería ayudar a los discentes a desarrollar su propio proceso de aprendizaje: «this guidance should not be provided by instructors as part of the assignment instruction but should be developed with the students through discussion and careful examination of the information qualities». Una utilización significativa o «high-level» de las TIC en el aula se asocia a prácticas constructivistas (Ertmer, 2005), que conllevan un cambio del papel del docente que pasa de tener la autoridad y el control del proceso formativo a ser un «referente cualificado» (Plaza De La Hoz, 2018). De hecho, diversos estudios (Gil-Flores, Rodríguez-Santero, \& Torres-Gordillo, 2017; Tirado-Morueta \& Aguaded-Gómez, 2014; Tondeur, Van Braak, Ertmer, \& Ottenbreit-Leftwich, 2017) concluyen que el profesorado que tiene una 
práctica pedagógica constructivista, centrada en el estudiante, hace un mayor uso de la tecnología en el aula.

Así pues, las creencias del docente sobre la naturaleza del conocimiento y sobre los procesos de enseñanza y aprendizaje son un factor que determina cómo utiliza las TIC en el aula (FragaVarela \& Rodríguez-Groba, 2017; C. Kim, Kim, Lee, Spector, \& Demeester, 2013; Prestridge, 2012) y esta relación es recíproca (Tondeur et al., 2017), es decir, el uso de las TIC en el aula modifica también la forma de trabajar del profesorado. Una revisión crítica de la literatura (Tondeur et al., 2017) señala que los docentes incorporan la tecnología dentro de los enfoques y estrategias metodológicas que ya poseen, sin abandonar su práctica docente habitual. No obstante, la presencia de la tecnología en el aula motiva al profesorado a experimentar y le hace avanzar hacia modelos educativos más constructivistas y centrados en el estudiante. En este mismo sentido, se infiere que «la presencia de las TIC obliga al profesor a replantearse cómo incorporarlas en su práctica y, en consecuencia, a realizar algún tipo de innovación con las mismas» (Area-Moreira, Hernández-Rivero, \& Sosa-Alonso, 2016, p. 85). En la misma línea, Hatlevik y Hatlevik (2018) constataron que la utilización de las TIC en e aula contribuyen, de forma indirecta, a que los profesores ayuden al alumnado a desarrollar su capacidad de evaluación de información digital.

Es necesario profundizar en el conocimiento de qué ocurre cuando se utilizan las TIC en los procesos de enseñanza y aprendizaje en el aula (Area-Moreira, Cepeda, González Salamanca, \& Sanabria, 2010; Coll, Mauri Majós, \& Onrubia Goñi, 2008). Mama y Hennessy (2013) observaron inconsistencias entre las opiniones y prácticas de los docentes: «many of the teachers' reports could be misleading, providing a 'false' picture of reality» (2013, p. 386). Asimismo, se ha comprobado que existe una distancia significativa entre el uso de las TIC previsto y el real (Coll et al., 2008). Por ello, inspirados en Cambra (2013), con esta investigación queremos contribuir a la identificación de las tensiones existentes entre el discurso y la práctica docente, por un lado, y contrastar el discurso y la práctica del profesorado con la del alumnado (Gil-Flores et al., 2017; Seguel, 2015), a partir de las interacciones que se construyen en el aula al realizar una actividad en la que se utiliza internet para buscar, evaluar y seleccionar información.

En relación a este objetivo, esta investigación se plantea responder a las siguientes preguntas: (a) ¿cuál es el discurso y las prácticas del alumnado al trabajar la búsqueda, evaluación y selección de información digital en el aula?, (b) ¿cuál es el discurso y las prácticas del profesorado al trabajar la búsqueda, evaluación y selección de información digital en el aula?

\section{Metodología}

Esta investigación es un estudio de caso (Merrian, 1998) desarrollado en un instituto de enseñanza secundaria del sur de Navarra durante el curso 2014-15 en la asignatura de Lengua Española y Literatura. Los estudios de caso se han mostrado como un enfoque válido para profundizar en la comprensión de la actividad didáctica relacionada con las TIC, tanto para medir el proceso como el grado de integración (Méndez Garrido \& Delgado García, 2016; Sancho Gil \& Padilla Petry, 2016), discernir sobre las estrategias utilizadas por el alumnado al leer en internet (Coiro \& Dobler, 2007) o conocer la opinión de alumnado y profesorado sobre su uso (Seguel, 2015). La elección del centro y de la asignatura estuvo motivada porque el Departamento de Lengua de este centro trabaja la asignatura de Lengua a través de proyectos organizados por medio de secuencias didácticas y la literatura señala que las estrategias de búsqueda, evaluación y selección de información no deben enseñarse de forma lineal y 
consecutiva, sino integradas dentro de un proyecto didáctico en el que el estudiante tenga la necesidad real de acudir a internet a buscar información (Kuiper, Volman, \& Terwel, 2009).

En la investigación participaron ocho profesores de Lengua Española y Literatura y un grupo de 24 estudiantes de 40 de la ESO, si bien no todos lo hicieron de la misma forma. Los ocho docentes tomaron parte de un grupo de discusión inicial que decidió centrar la investigación en un proyecto de $4^{\circ}$ de la ESO en el que el alumnado tiene que documentarse buscando información en internet para realizar un reportaje periodístico. En años anteriores, se había llevado a cabo este dispositivo didáctico, pero la búsqueda de información en internet era una tarea que el alumnado había realizado como deberes escolares en casa, sin el apoyo del profesor. Cuatro profesores de $4^{\circ}$ de la ESO llevaron al aula el nuevo dispositivo didáctico, tras participar en un proceso de formación en el que modificaron la secuencia didáctica (Dolz \& Gagnon, 2010) introduciendo estrategias relacionadas con la planeación, búsqueda, evaluación y selección de información:

- Estrategias de planeación (elección de tema, realización de preguntas sobre el tema, formulación de objetivos de búsqueda, diferenciación de fuentes primarias y secundarias)

- Estrategias de búsqueda, evaluación, selección y gestión de fuentes digitales (utilización de palabras claves, búsquedas avanzadas en Google, reformulación de palabras claves, gestión de páginas web)

- Estrategias de evaluación de fuentes y de información (plantilla para la evaluación de las fuentes y de la información)

- Estrategias de selección de información (extracción de ideas principales, comparación y selección de información)

Para mejorar la validez de los resultados, se ha diseñado una triangulación de datos multifuente (Rodríguez Ruiz, 2005) en la que se recaban datos tanto del alumnado como de sus profesores (Ver Tabla 1). La fuente principal de información es la actividad desarrollada en el aula. Esta se complementa con las reflexiones narradas por los propios actores en grupos de discusión (Murillo \& Mena, 2006) y a través de la técnica de autoconfrontación (Thereau, 2010). A través de esta triangulación de datos se persigue la compresión de los fenómenos estudiados y contrastar el discurso y la práctica tanto de los docentes como de los discentes.

Se registraron 6 sesiones de uno de los profesores de $4^{\circ}$ de la ESO que trabajó con el alumnado en al aula la búsqueda, evaluación y selección de información y, durante estas, se utilizó el programa Camtasia para capturar el trabajo realizado en el ordenador de tres parejas de alumnos. Estos seis estudiantes son los que participaron en el grupo de discusión final. Se editó un vídeo a partir de las grabaciones de las clases y del trabajo registrado de las tres parejas de estudiantes para las entrevistas de autoconfrontación con el profesor cuyas sesiones fueron grabadas (autoconfrontación simple) y con los cuatro profesores de $4^{\circ}$ de la ESO que llevaron al aula la secuencia didáctica (autoconfrontación grupal). Esta técnica da al profesorado la oportunidad de reflexionar sobre su práctica a partir del visionado de un vídeo en el que esta haya sido registrada. 


\begin{tabular}{|c|c|c|}
\hline & $\begin{array}{l}\text { Técnicas de recogida } \\
\text { de datos }\end{array}$ & Descripción \\
\hline \multirow[b]{2}{*}{$\begin{array}{l}0 \\
\text { U } \\
\text { U } \\
\\
\end{array}$} & $\begin{array}{l}\text { Grupo de discusión con } \\
\text { el alumnado inicial } \\
\text { (GDAi) }\end{array}$ & $\begin{array}{l}\text { Participaron } 8 \text { estudiantes y se indagó sobre el uso que hacían } \\
\text { de las TIC tanto dentro como fuera del aula así como sobre su } \\
\text { opinión sobre estos usos. }\end{array}$ \\
\hline & $\begin{array}{l}\text { Grupo de discusión con } \\
\text { el profesorado inicial } \\
\text { (GDPi) }\end{array}$ & $\begin{array}{l}\text { Participaron } 8 \text { docentes del Departamento de Lengua. En el } \\
\text { grupo de discusión se abordó cómo trabajaban en el aula la } \\
\text { búsqueda, evaluación y selección de información y su } \\
\text { percepción sobre la actividad del alumnado, y se decidió en qué } \\
\text { grupo y en qué curso centrar la investigación. }\end{array}$ \\
\hline \multirow{2}{*}{ 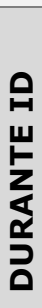 } & $\begin{array}{l}\text { Videograbación de las } \\
\text { sesiones }(\mathrm{S} 1, \mathrm{~S} 2 \ldots \mathrm{S} 6)\end{array}$ & $\begin{array}{l}\text { Se registraron seis sesiones en las que un grupo de } 24 \\
\text { estudiantes trabajó la búsqueda, evaluación y selección de } \\
\text { información digital. }\end{array}$ \\
\hline & $\begin{array}{l}\text { Trabajo del alumnado } \\
\text { en el aula (TA) }\end{array}$ & $\begin{array}{l}\text { Con el programa Camtasia se registró el trabajo de tres parejas } \\
\text { de estudiantes }(n=6) \text {, elegidas por el docente, durante las } \\
\text { sesiones que trabajaron con ordenadores }(S 2, S 3, S 4 \text { Y S6). }\end{array}$ \\
\hline \multirow{3}{*}{ 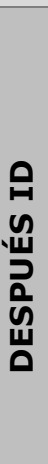 } & $\begin{array}{l}\text { Grupo de discusión con } \\
\text { el alumnado final } \\
\text { (GDAf) }\end{array}$ & $\begin{array}{l}\text { Participaron las tres parejas de estudiantes registradas }(n=6) \text { y } \\
\text { se habló sobre sus prácticas, buscando profundizar en la } \\
\text { comprensión de su actividad. }\end{array}$ \\
\hline & $\begin{array}{l}\text { Entrevista de } \\
\text { autoconfrontación (AC) }\end{array}$ & $\begin{array}{l}\text { Se confrontó al profesor con un vídeo en el que se presentaron } \\
\text { episodios de su actividad a lo largo de las seis sesiones. }\end{array}$ \\
\hline & $\begin{array}{l}\text { Entrevista } \\
\text { autoconfrontación } \\
\text { grupal (ACG) }\end{array}$ & $\begin{array}{l}\text { El profesor cuyas clases fueron grabadas junto a otros tres } \\
\text { docentes que llevaron al aula la misma secuencia didáctica } \\
\text { ( } n=4 \text { ) fueron confrontados a un vídeo en el que se presentaron } \\
\text { episodios del grupo que se registró. }\end{array}$ \\
\hline
\end{tabular}

Tabla. 1. Instrumentos de investigación usados antes, durante y después de la intervención didáctica y acrónimos utilizados en el texto.

Para el análisis de datos, tanto los grupos de discusión como de la actividad en el aula y las autoconfrontaciones, utilizamos las normas y estándares de competencia en alfabetización informacional (American Library Association, 2000). La American Library Association (ALA) define cinco normas generales que se desglosan en un total de veintitrés estándares de rendimiento. En nuestro análisis tuvimos en cuenta las tres primeras normas y los estándares correspondientes relacionados con nuestro objeto de estudio que se encuentra en la columna de la izquierda de la Tabla 2. A partir de sucesivas lecturas de las transcripciones, se establecieron las categorías de análisis al identificar las instrucciones docentes en las que se reflejaban las normas APA (en la columna derecha de la Tabla 2). Encontramos que algunas prácticas del alumnado, tales como distracciones o pérdida de tiempo, no aparecían ni en las normas APA ni en la instrucción docente, pero nos parecía interesante marcarlas en el análisis. Por esta razón, se crearon nuevas categorías de análisis (marcadas en cursiva en la columna de la derecha de la Tabla 2). De esta forma, conseguimos un marco de análisis que nos permitió un acercamiento y una comprensión más profunda de la actividad didáctica del aula. 


\begin{tabular}{|c|c|}
\hline $\begin{array}{l}\text { Normas y estándares de alfabetización } \\
\text { información de la American Library } \\
\text { Association }\end{array}$ & $\begin{array}{l}\text { Categorías de análisis a partir de la concreción de } \\
\text { los estándares ALA en la instrucción docente } \\
\text { Categorías emergentes }\end{array}$ \\
\hline \multicolumn{2}{|c|}{$\begin{array}{l}\text { NORMA 1: El estudiante es capaz de determinar la naturaleza y nivel de la información que } \\
\text { necesita. }\end{array}$} \\
\hline \multirow{3}{*}{$\begin{array}{l}\text { Define y articula sus necesidades de } \\
\text { información. }\end{array}$} & Elegir un tema para el trabajo. \\
\hline & $\begin{array}{l}\text { Identificar objetivos de búsqueda, preguntas sobre } \\
\text { el tema. }\end{array}$ \\
\hline & Desinterés por el tema \\
\hline \multirow[t]{2}{*}{$\begin{array}{l}\text { Identifica varios tipos y formatos de } \\
\text { información. }\end{array}$} & $\begin{array}{l}\text { Identificar y diferenciar el valor de fuentes } \\
\text { primarias (entrevista) y secundarias (internet, } \\
\text { documentos). }\end{array}$ \\
\hline & $\begin{array}{l}\text { Preferencia por la información en formato } \\
\text { audiovisual. }\end{array}$ \\
\hline Se replantea la información que necesita. & Revisar objetivos de búsqueda. \\
\hline \multicolumn{2}{|c|}{$\begin{array}{l}\text { NORMA 2: El estudiante es capaz de acceder a la información requerida de manera eficaz y } \\
\text { eficiente. }\end{array}$} \\
\hline \multirow{2}{*}{$\begin{array}{l}\text { Selecciona sistemas adecuados de } \\
\text { recuperación de la información. }\end{array}$} & Utilizar Google como buscador \\
\hline & Utiliza otros buscadores (Youtube...) \\
\hline \multirow{3}{*}{$\begin{array}{l}\text { Pone en práctica estrategias eficaces de } \\
\text { búsqueda. }\end{array}$} & Utilizar palabras clave adecuadas \\
\hline & Utilizar opciones de Búsqueda Avanzada \\
\hline & $\begin{array}{l}\text { Desinterés por la búsqueda de información en } \\
\text { internet }\end{array}$ \\
\hline Refina la estrategia de búsqueda. & Añadir o reformular palabras clave \\
\hline \multicolumn{2}{|c|}{$\begin{array}{l}\text { NORMA 3: El estudiante evalúa la información y sus fuentes de forma crítica e incorpora la } \\
\text { información seleccionada a su propia base de conocimientos y a su sistema de valores. }\end{array}$} \\
\hline Resume las ideas principales & Leer el texto y seleccionar las ideas principales. \\
\hline \multirow{7}{*}{$\begin{array}{l}\text { Aplica criterios para evaluar la información } \\
\text { y sus fuentes. }\end{array}$} & Evaluar la claridad y comprensibilidad \\
\hline & Evaluar la validez de la información \\
\hline & Evaluar la autoría y fiabilidad \\
\hline & Evaluar el punto de vista o sesgo ideológico \\
\hline & Evaluar la intención \\
\hline & Evaluar la vigencia o actualidad \\
\hline & $\begin{array}{l}\text { Desinterés por la evaluación de la información y } \\
\text { sus fuentes }\end{array}$ \\
\hline \multirow{2}{*}{$\begin{array}{l}\text { Compara los nuevos conocimientos con los } \\
\text { anteriores. }\end{array}$} & Sacar conclusiones de la información obtenida. \\
\hline & Comparar la información de diferentes fuentes. \\
\hline
\end{tabular}




\begin{tabular}{|l|l|}
\hline $\begin{array}{l}\text { Determina el impacto del nuevo } \\
\text { conocimiento en sus valores. }\end{array}$ & $\begin{array}{l}\text { Decidir si incorpora o rechaza los puntos de vista } \\
\text { encontrados. }\end{array}$ \\
\cline { 2 - 3 } & Impacto emocional de los contenidos. \\
\hline $\begin{array}{l}\text { Valida la comprensión por medio de } \\
\text { intercambio de opiniones. }\end{array}$ & $\begin{array}{l}\text { Construir la compresión de la información en la } \\
\text { interacción entre pares. }\end{array}$ \\
\cline { 2 - 2 } & $\begin{array}{l}\text { No colabora u obstaculiza el trabajo del } \\
\text { compañero/a. }\end{array}$ \\
\hline Distracciones & $\begin{array}{l}\text { Pérdida de tiempo en conversaciones o búsquedas } \\
\text { que no tienen relación con su trabajo. }\end{array}$ \\
\hline
\end{tabular}

Tabla. 2. Categorías de análisis a partir de las normas y estándares de alfabetización informacional, modificadas a partir de la instrucción docente y la práctica discente.

Una vez transcritas las grabaciones de las sesiones de trabajo, se procedió a su segmentación en unidades de análisis con el objetivo de facilitar su estudio. Finalmente, para llegar a los resultados que se presentan a continuación, se puso en relación el análisis de la actividad desarrollada por el alumnado en el aula con la información obtenida a través de las autoconfrontaciones y los grupos de discusión del alumnado y del profesorado. Se realizó una codificación cruzada ciega entre tres investigadores.

\section{Resultados}

En cuanto a la capacidad del alumnado para definir y articular sus necesidades de información, encontramos que algunos estudiantes tienen problemas a la hora de elegir el tema. La razón de esto puede ser que, según expusieron los estudiantes, el profesorado normalmente les proporciona los temas de los trabajos. No obstante, una alumna que hace el trabajo sobre un tema propuesto por el profesor muestra desinterés por el tema, señala que lo ha hecho «medio obligada» y tiene numerosas dificultades en el proceso. Aquellos que eligen temas de los que tienen conocimientos previos y que pueden relacionar con su vida tienen menos dificultades en el proceso de búsqueda: «Rocío: pero es mejor un tema que más o menos conozcas, porque así ya vas..., no sé así se te hace más fácil» (GDAf). Los estudiantes que trabajan sobre temas con los que no están familiarizados (desahucios, yihadismo) tienen más problemas en el proceso de búsqueda. Solo dos alumnas planifican por escrito los aspectos del tema sobre los que quieren buscar información, pero una de ellas fija 16 objetivos de búsqueda, lo que resulta excesivo para la dimensión del trabajo a realizar. El profesorado detecta que el alumnado, con algunas excepciones, tiene dificultades para determinar los objetivos de búsqueda de información y que necesitan andamiaje para ello: «Juan: el problema es que les cuesta mucho elegir por dónde van a tirar, dentro de ese tema, qué es lo que les interesa (...) les cuesta mucho ese primer paso, antes incluso que buscar» (ACG).

En relación a los tipos y formatos de información que identifican, los estudiantes, inicialmente, muestran gran entusiasmo por trabajar en clase conectados a internet y manifiestan que internet es una fuente de información más rápida, fácil y entretenida que los libros o incluso que las explicaciones del profesor: «Juan: [trabajar con internet en clase] sería la hostia» (GDAi) «Lorea: [con internet] haces los trabajos muchísimo más rápido y a veces se entiende muchísimo mejor que un libro o un profesor» (GDAi). Sin embargo, durante el trabajo en el aula, una alumna demuestra gran desinterés por el trabajo y dos alumnas manifiestan que es mejor y más fácil obtener la información de de una fuente humana que en internet: «Carolina: 
Búsqueda, evaluación y selección de información digital en un aula de secundaria: Tensiones entre la práctica y el discurso del

buscar en internet vale mucho, pero hacer la entrevista también porque la información que te aporta la persona que estás preguntando creo que es mucho más completa que la puedes encontrar en internet» (GDAf). Con internet, el alumnado percibe que existe riesgo de distracción: «Carlos: está el riesgo de descentrarte y bajará la media porque, claro, no pondrás atención en clase por estar con tus cosas en internet» (GDAf). El profesorado ve internet como una fuente más de documentación, entre otras, y da preponderancia a la información escrita. Ante el temor de que el alumnado no encuentre información fiable, opta por darle las URLs de las páginas. Sin embargo, todos los grupos de estudiantes buscan información en modalidades tales como imágenes, vídeos y gráficos, mostrando preferencia por la información audiovisual: «Mikel: pero es que con una foto no hacemos nada; es preferible ver un vídeo en Youtube de lo que es un yihadista, que se diga cómo es y ya está (+Nueva pestaña; escribe en la barra de búsqueda de Google youtube.com; + Abrir» (TA).

En torno a la selección de sistemas adecuados de recuperación de información y la puesta en práctica de estrategias eficaces de búsqueda, el alumnado reconoce que, en ocasiones, no encuentra la información que buscan: «Rocío: no sé, igual te salen muchas cosas que no te sirven o que... tú tampoco te expresas bien al poner lo quieres buscar, entonces el ordenador no te busca lo que tú quieres» (GDAf). Los estudiantes señalan Wikipedia como una de sus fuentes principales de información a la hora de realizar trabajos para la escuela. La práctica así lo confirma, pues todos los grupos utilizan la enciclopedia virtual para un primer acercamiento al tema. Asimismo, todos utilizan la plataforma de YouTube para buscar vídeos y la opción de Imágenes de Google para acceder a gráficos. Aquellos que trabajan sobre un tema que conocen acuden a páginas con la que ya están familiarizados. Cuando el profesorado explicó cómo utilizar la herramienta de Búsqueda Avanzada de Google, una profesora se vio muy sorprendida porque algunas de sus alumnas hicieron búsquedas por colores, aspecto que ella desconocía. Alumnado y profesorado coinciden al reconocer la importancia de los conocimientos previos a la hora de la búsqueda de información y varios estudiantes señalan que no se pone el mismo interés en la búsqueda si se trata de un tema personal o de una tarea escolar: «Daniel: si es para ti, buscas más; si es para la escuela, probablemente te quedas con lo primero que ves» (GDAf). El profesorado, por su parte, manifiesta la necesidad de dirigir la búsqueda proporcionándoles las URLs: «Miguel: (...)yo creo que el alumno, sin el conocimiento previo sobre el tema, se encontraba ante un reto inmenso (...) por eso, la búsqueda de información tiene que ser siempre dirigida: nosotros tenemos que buscar las páginas donde ellos se han de centrar en su trabajo, si no eso es un bosque inextricable» (GDP).

A la hora de replantearse la información que necesitan y de refinar las estrategias de búsqueda, algunas estudiantes muestran que tienen presente el objetivo de la búsqueda a la hora de seleccionar la entrada de información: «Rocío: iah!, pero ¿te quieres alojar ahí? ¿quieres que busque información para alojarnos ahí o en plan cómo se fundaron y todo eso? (hace clic en la ENTRADA Casas Cueva de Las Bardenas Reales: lasbardenas.com)» (TA). Durante la búsqueda, la mayoría de estudiantes reformulan los términos acotándolos geográfica y temporalmente. Aquellos discentes que tienen escasos conocimientos previos sobre el tema tienen más dificultades para reformular los términos de búsqueda. Es el caso del estudiante que busca información sobre yihadismo y que solo utiliza la palabra clave «yihadismo» para todas sus búsquedas. Algunos alumnos señalan que reformulan sus objetivos de búsqueda a partir de los resultados obtenidos: «Carolina: cuando te enteras un poco más del tema, tienes, como dice Rocío, más ideas para buscar otras cosas» (GDAf). El profesorado, en general, considera que el alumnado tiene problemas para hacer búsquedas específicas.

En cuanto a los criterios de evaluación de la información y de sus fuentes, encontramos que aunque el alumnado dice saber que en internet hay información falsa, en general, no presta 
atención a la autoría ni al sesgo ideológico de la información que consultan: «Alba: yo no me fijo (en la autoría), total, si tiene la información que estás buscando, ¿para qué quieres saber quién es el autor?» (GDAf) «Fermín: ¿a qué te refieres con eso del autor?» (GDAf). Al evaluar la comprensibilidad de la información, los estudiantes exponen que, en muchas ocasiones, tienen dificultades para la comprensión de la información que aparece en Wikipedia «Ander: la Wikipedia da asco (...) no te aclara nada la Wikipedia (...) copias y pegas y ya está, pero no te sirve para entender nada porque pone en un párrafo un montón de palabras que es que no se entienden» (GDAf). Solo un alumno que tiene una postura definida sobre su tema de búsqueda, la caza, y que durante la búsqueda se da cuenta que una de las páginas que consulta es contraria a sus ideas, reflexiona sobre la intención y el sesgo ideológico de la información; el resto no lo hace. La mayoría valora mejor las páginas que tienen gran cantidad información. También evalúan la actualidad de la información que consultan. Respecto a la credibilidad, el alumnado percibe que el profesorado no tiene confianza en la información que aparece en la red: «Leire: no tienes la certeza de que sea verdad. Por eso, muchas veces los profesores buscas algo en internet y luego dicen 'esto has sacado de internet porque esto no es asi'. Confías en lo que sale y ves que no» (GDAi). Los docentes, por otro lado, contemplan la red como un entorno amenazante, «un bosque inextricable», y consideran que el alumnado no es capaz de evaluar y seleccionar información adecuada en la red, por lo que optan por restringirles el tipo de páginas a consultar.

Respecto a la forma en que el alumnado trabaja con el contenido de la información, este manifiesta que su práctica más habitual es básicamente copiar y pegar la información que aparece en internet, en ocasiones realizando pequeñas modificaciones: «Carlos: yo cojo una página y ya está, y si eso, pues cambio alguna palabra por sinónimos y ya está» (GDAi) "Rocío: leía toda la página, veía lo más importante, lo copiaba en el Word y así con todas" (GDAf) El profesorado es consciente de esta práctica y considera que puede ser válida si luego el alumnado es capaz de construir un texto cohesionado y siempre que cite la procedencia de la información. La mayoría de alumnos y alumnas identifica las ideas principales del texto a partir de la lectura de los titulares y epígrafes y, normalmente, se queda con la información que aparece al inicio del texto. En general, tienen dificultades a la hora de extraer información de modalidades textuales diversas: gráficos y vídeos. Las tres parejas de estudiantes tienen problemas para interpretar los gráficos a los que, la mayor parte de las veces, acceden de forma descontextualizada, a través de la opción de Imágenes de Google. Respecto a los vídeos, en ocasiones, se quedan con información superficial como, por ejemplo, el aspecto de las personas que aparecen: «Alba: (viendo un vídeo) este tiene pintas de malote, este va de soso, este va de lo sé todo cállate» (TA). Dos de las parejas de estudiantes acceden a contenidos violentos, que les impacta emocionalmente, especialmente el grupo que visualiza escenas de violencia extrema en un contexto de guerra quedan marcados por el impacto emocional de las imágenes: «Mikel: esto no es normal tío (hace clic en un vídeo que aparece en el lateral VÍDEO Soldados capturados e torturados por extremista do Estado Islámico \{sale un hombre en el suelo con el torso descubierto y marcas de golpes\} voy a quitar eso que es que no lo puedo ni ver» (TA). El profesorado considera que a los estudiantes les cuesta relacionar la información procedente de fuentes diversas y que necesita apoyo para hacerlo.

También hemos analizado cómo se validaba entre pares la comprensión de la información y las posibles distracciones durante la tarea. Hemos observado que aunque el alumnado trabaja en parejas, no siempre lo hace de forma cooperativa. Los discentes consideran que, entre pares, se aprende más y que el trabajo en parejas resulta más entretenido «Lorea: siempre hay compañeros que tienen más perrerías que otro y le preguntas cómo lo ha hecho y ya lo ves y lo haces" (GDAi) «Ander: si estás solo, quieres acabar más rápido, coges lo primero que pillas y acabas. Si estás con un compañero es más divertido y quieres estar más tiempo» (GDAf). En 
Búsqueda, evaluación y selección de información digital en un aula de secundaria: Tensiones entre la práctica y el discurso del

ocasiones, construyen conocimiento mediante la interacción, pero, en una de las parejas, una de alumnas es la que realiza todo el trabajo ante el pasotismo y desinterés de su compañera por buscar información en la red. $Y$ en la tercera, encontramos un trabajo desigual entre los miembros y, en ocasiones, se llega a boicotear el trabajo del compañero. «Daniel: eres tonto o qué/ eres subnormal/ va el tío y me quita el pendrive (...) a la próxima que me hagáis algo os juro que os reviento» (TA).

\section{Conclusiones y discusión}

Al tratarse de un estudio de caso, sus conclusiones no son generalizables a todos los centros. No obstante, consideramos que esta investigación contribuye significativamente a la comprensión de la actividad didáctica sobre la búsqueda, evaluación y selección de información digital y puede ser útiles al planificar la integración de las TIC en los centros de secundaria.

\section{a. Discurso y prácticas del alumnado}

La mayor parte del alumnado, como se ha visto en otros estudios (Gialamas et al., 2013), muestra interés y preferencia por trabajar con internet en el aula. Sin embargo, percibe que el profesorado no confía en la información que aparece en la red y que resta legitimidad a internet como fuente de información. Asimismo, algunos alumnos exponen el riesgo de distracción que existe al usar internet y cómo puede influir negativamente en sus resultados académicos. Esta visión, en sintonía con lo que concluye Campos Martínez (2015), pudiera estar influenciada por la opinión de los adultos. En este sentido, podemos inferir que las creencias del profesorado sobre las TIC y su uso en el aula no solo influyen en su práctica sino que también pueden condicionar la propia percepción del alumnado.

A pesar de que el alumnado manifiesta un gran interés por trabajar con internet en clase, en la práctica encontramos estudiantes con gran apatía ante la tarea de búsqueda de información. Ellos mismos reconocen que tienen una actitud diferente al buscar información que responda a intereses personales a si lo hacen para una tarea escolar. Trabajar partiendo de los intereses del alumnado y de problemas auténticos que conlleven una fuerte carga emocional del alumnado facilita su implicación en la tarea (Monereo \& Badia, 2012). Además, se ha visto que en línea con estudios previos (Amadieu, Tricot, \& Mariné, 2009; Coiro \& Dobler, 2007), los conocimientos previos sobre el tema facilitan el proceso de búsqueda. Aquellos que no están familiarizados con el tema (yihadismo, desahucios) tienen dificultades en la selección de páginas y a la hora de evaluar y seleccionar la información. Este es un dato relevante a la hora de abordar la enseñanza de la búsqueda de información en línea, ya que dependiendo de los objetivos y contextos de búsquedas se debería trabajar en el aula diferentes estrategias de búsqueda.

La linealidad de las normas y estándares de alfabetización informacional no se corresponde con la complejidad, recursividad e iteración que conlleva el proceso de búsqueda real. La mayoría del alumnado no establece objetivos de búsqueda de información y va acotando y redefiniendo la búsqueda a partir de los resultados que encuentra. Sería interesante seguir estudiando qué es lo que hace que algunos alumnos sean más competentes que otros en la redefinición de objetivos de búsqueda y cómo desarrollar una competencia metacognitiva sobre la búsqueda de información más cercana al modelo berrypicking que a una estrategia lineal y planificada (Egaña, Bidegain, \& Zuberogoitia, 2013). 
Aunque algunas investigaciones apuntan que el alumnado utiliza Wikipedia por su accesibilidad y comprensibilidad (Valverde-Crespo \& González-Sánchez, 2016), en esta investigación, si bien la utilizan, los discentes han manifestado que tiene problemas en la comprensión de la información que ofrece Wikipedia. También hemos observado que tienen importantes dificultades para la comprensión de gráficos y para la interpretación y apropiación de la información audiovisual. No parece fácil trasladar la información audiovisual a la modalidad escrita (Sevensma, 2013), por lo que consideramos que es necesario empezar a trabajar en el aula la transmediación de información entre diferentes modalidades textuales (Smith, Kiili, \& Kauppinen, 2016).

Además, al alumnado le cuesta entender los conceptos de autor y plagio y para evaluar el sesgo ideológico de la información que consulta. Es imprescindible que desarrolle una actitud crítica (Sancho Gil \& Padilla Petry, 2016) ante una globosfera comunicativa que no distingue al receptor de la información y donde cada vez hay mayor cantidad de datos y contaminación informativa. La evaluación de la información de internet y sus fuentes es uno de los grandes retos educativos.

\section{b. Discurso y prácticas del profesorado}

Los docentes, en general, consideran que internet es un «bosque inextricable» y que los estudiantes no son capaces de buscar información adecuada en internet por su cuenta, por lo que la mayoría les proporciona las URL que deben consultar o les orientan hacia un determinado tipo de páginas. Esta práctica impide que el alumnado experimente en un entorno de aprendizaje con búsquedas abiertas. Resulta recomendable que el profesor realice una transferencia progresiva del control de la actividad hacia el alumno (Hernández Serrano \& Fuentes Agustí, 2011), con el objetivo de que desarrolle una actitud crítica en una navegación abierta en la red.

Se ha evidenciado que los discentes necesitan apoyo y andamiaje para la selección de tema, para establecer y redefinir los objetivos de búsqueda y para la evaluación y selección de información, y el profesorado se muestra consciente de ello; sin embargo, no siempre lo trabaja en el aula. Es importante dotar al profesorado de los recursos necesarios y proporcionarle una formación adecuada que aborde también las creencias pedagógicas para que lo integre en su labor (Méndez Garrido \& Delgado García, 2016). Para incidir en estas creencias se muestra más efectiva una formación a largo plazo, organizada como proceso iterativo en el contexto de trabajo, apoyada en comunidades de práctica y en el trabajo colaborativo entre docentes (Tondeur et al., 2017).

El trabajo con internet en el aula cuestiona el papel del profesor como poseedor único del conocimiento y los docentes expresan su temor a perder el control de la clase por posibles problemas técnicos o por el hecho de que surjan situaciones a las que no sepan cómo responder. Un trabajo con TIC en el aula, en el que se da el protagonismo al alumnado, conlleva un cambio de rol del profesorado, que debe reconocer sus limitaciones y hacer ver al alumnado las suyas (Plaza de la Hoz, 2018) para avanzar hacia estructuras de autoridad más horizontales (Campos Martínez, 2015).

Por último, hemos observado situaciones de trabajo desigual en los grupos de estudiantes e incluso de boicot entre pares, lo que deja en evidencia que para que se dé un trabajo colaborativo no es suficiente trabajar en grupo. Aunque se ha comprobado que el aprendizaje colaborativo con TIC contribuye a la construcción del conocimiento (García- 
Búsqueda, evaluación y selección de información digital en un aula de secundaria: Tensiones entre la práctica y el discurso del

Valcárcel, Basilotta, \& López, 2014), es necesario tomar en cuenta algunos aspectos como la familiaridad entre los componentes de grupo, la complejidad de la tarea en relación a roles de los participantes o un rol activo del docente para que se cree una dinámica colaborativa (Avello-Martínez \& Marín, 2016).

Este estudio tiene las limitaciones propias de un estudio de caso, por lo que es necesario seguir investigando para lograr la comprensión cabal de una realidad compleja como la educativa. Con el fin de facilitar al profesorado su labor, es importante determinar con mayor precisión los factores que intervienen en la interacción didáctica cuando se trabaja la búsqueda, evaluación y selección de información digital. Se trata de avanzar hacia un modelo educativo que tome en cuenta las necesidades y prácticas vernáculas del alumnado en una sociedad donde la alfabetización informacional cobra cada vez más relevancia.

\section{Referencias}

Amadieu, F., Tricot, A., \& Mariné, C. (2009). Prior knowledge in learning from a non-linear electronic document: Disorientation and coherence of the reading sequences. Computers in Human Behavior, 25(2), 381-388. doi: https://doi.org/10.1016/j.chb.2008.12.017

American Library Association. (2000). Information Literacy Competency Standards for Higher Education. doi: http://doi.org/efeb57df-7090-e1d4-558f-d59c7537f9c7

Area-Moreira, M., Cepeda, O., González Salamanca, D., \& Sanabria, A. (2010). Un análisis de las actividades didácticas con TIC en aulas de Educación Secundaria. Pixel-Bit: Revista de Medios y Educación, 38, 187-199.

Area-Moreira, M., Hernández-Rivero, V., \& Sosa-Alonso, J.-J. (2016). Modelos de integración didáctica de las TIC en el aula. Comunicar, 47, 79-87. doi: https://doi.org/10.3916/C47-2016-08

Avello-Martínez, R., \& Marín, V. I. (2016). La necesaria formación de los docentes en aprendizaje colaborativo. Profesorado. Revista de Currículum y Formación Del Profesorado, 20(3), 687-713.

Biddix, J. P., Chung, C. J., \& Park, H. W. (2011). Convenience or credibility? A study of college student online research behaviors. The Internet and Higher Education, 14(3), 175-182. doi: https://doi.org/10.1016/J.IHEDUC.2011.01.003

Cambra, M. (2013). Investigar la interacción en las clases de lenguas. Cultura \& Educación, 25(4), 429-440.

Campos Martínez, J. A. (2015). Lo cotidiano (entre usos y resistencias) de las TIC en un aula de Educación Secundaria Obligatoria. Antropología Experimental, O(15), 567-580.

Coiro, J., \& Dobler, E. (2007). Exploring the online reading comprehension strategies used by sixth-grade skilled readers to search for and locate information on the Internet. Reading Research Quarterly, 42(2), 214-257. doi: https://doi.org/10.1598/RRQ.42.2.2

Coll, C., Mauri Majós, M. T., \& Onrubia Goñi, J. (2008). Análisis de los usos reales de las TIC en contextos educativos formales: una aproximación socio-cultural. Revista Electrónica de Investigacion Educativa, 10(1), 1-18. doi: https://doi.org/ISSN 1607-4041

Dolz, J., \& Gagnon, R. (2010). El género textual, una herramienta didáctica para desarrollar el lenguaje oral y escrito. Lenguaje, 38(2), 497-527.

Eagleton, M., Guinee, K., \& Langlais, K. (2003). Teaching Internet literacy strategies: The hero inquiry project. Voices From the Middle, 10(3), 28-35.

Egaña, T., Bidegain, E., \& Zuberogoitia, A. (2013). ¿Cómo buscan información académica en internet los estudiantes universitarios? Lo que dicen los estudiantes y sus profesores. Edutec: Revista Electrónica de Tecnología Educativa, 43, 1-15.

Ertmer, P. A. (2005). Teacher pedagogical beliefs: The final frontier in our quest for technology integration? Educational Technology Research and Development, 53(4), 25-39. doi: https://doi.org/10.1007/BF02504683 
Búsqueda, evaluación y selección de información digital en un aula de secundaria: Tensiones entre la práctica y el discurso del profesorado y el alumnado

Fraga-Varela, F., \& Rodríguez-Groba, A. (2017). Dilemas y desafios de la Tecnología Educativa en el EEES: Percepciones y creencias de futuros maestros. Profesorado. Revista de Currículum y Formación Del Profesorado, 21(1), 123-142.

Fraga Varela, F., \& Gewerc Barujel, A. (2013). Creencias sobre Tecnología Educativa de profesorado en formación: un estudio de caso. Innovación Educativa, 23, 241-254.

Gairín, J., \& Mecader, C. (2018). Usos y abusos de las TIC en adolescentes. Revista de Investigación Educativa, 36(1), 125-140. doi: https://doi.org/http://dx.doi.org/10.6018/rie.36.1.284001

García-Valcárcel, A., Basilotta, V., \& López, C. (2014). Las TIC en el aprendizaje colaborativo en el aula de Primaria y Secundaria. Comunicar, 21(42), 1-8. doi: https://doi.org/http://dx.doi.org/10.3916/C422014-06 Las

Gialamas, V., Nikolopoulou, K., \& Koutromanos, G. (2013). Student teachers' perceptions about the impact of internet usage on their learning and jobs. Computers \& Education, 62, 1-7. doi: https://doi.org/10.1016/J.COMPEDU.2012.10.012

Gil-Flores, J., Rodríguez-Santero, J., \& Torres-Gordillo, J. J. (2017). Factors that explain the use of ICT in secondary-education classrooms: the role of teacher characteristics and school infrastructure. Computers in Human Behavior, 68, 441-449. doi: https://doi.org/10.1016/j.chb.2016.11.057

Hatlevik, I. K. R., \& Hatlevik, O. E. (2018). Students' evaluation of digital information: The role teachers play and factors that influence variability in teacher behaviour. Computers in Human Behavior, 83, 56-63. doi: https://doi.org/10.1016/j.chb.2018.01.022

Hernández Serrano, M. J., \& Fuentes Agustí, M. (2011). Aprender a informarse en la red: ¿son los estudiantes eficientes buscando y seleccionando información? TESI, 12(1), 47-78.

Kim, C., Kim, M. K., Lee, C., Spector, J. M., \& Demeester, K. (2013). Teacher beliefs and technology integration. Teaching and Teacher Education, 29(1), 76-85. doi: https://doi.org/10.1016/j.tate.2012.08.005

Kim, K.-S., \& Sin, S.-C. J. (2011). Selecting quality sources: Bridging the gap between the perception and use of information sources. Journal of Information Science, 37(2), 178-188.

Kirschner, P. A., \& Bruyckere, P. De. (2017). The myths of the digital native and the multitasker. Teaching and Teacher Education, 67, 135-142. doi: https://doi.org/10.1016/j.tate.2017.06.001

Kuiper, E., Volman, M., \& Terwel, J. (2009). Developing Web literacy in collaborative inquiry activities. Computers \& Education, 52(3), 668-680. doi: https://doi.org/10.1016/j.compedu.2008.11.010

Mama, M., \& Hennessy, S. (2013). Developing a typology of teacher beliefs and practices concerning classroom use of ICT. Computers \& Education, 68, 380-387. doi: https://doi.org/10.1016/j.compedu.2013.05.022

Méndez Garrido, J. M., \& Delgado García, M. (2016). Las TIC en centros de Educación Primaria y Secundaria de Andalucía. Un estudio de casos a partir de buenas prácticas. Digital Education Review, 29, 134165.

Merrian, S. B. (1998). Qualitative Research and Case Study Applications in Education. San Francisco: Jossey-Bass.

Monereo, C., \& Badia, A. (2012). La competencia informacional desde una perspectiva psicoeducativa: enseñanza basada en la resolución de problemas prototípicos y emergentes. Revista Española de Documentación Científica, 35(Monográfico), 75-99.

Montoya Camacho, M. J. (2017). La era de la posverdad, la posveracidad y la charlatanería. Palabra, 5659.

Murillo, S., \& Mena, L. (2006). Detectives y camaleones: el grupo de discusión. Una propuesta para la investigación cualitativa. Madrid: Talasa.

Plaza De La Hoz, J. (2018). Autoridad docente y Nuevas Tecnologías: cambios, retos y oportunidades. Revista Complutense de Educación, 29(1), 269-286. doi: https://doi.org/10.5209/RCED.52281

Pow, J., \& Li, C. S. (2015). The effect of students' perceptions of Internet information quality on their use of Internet information in inquiry-based learning. Australasian Journal of Educational Technology, 31(4), 439-457.

Pow, J. W. C., Li, S. C., \& Fung, A. C. W. (2009). Students' inquiry learning in the web 2.0 age. In C. 
Búsqueda, evaluación y selección de información digital en un aula de secundaria: Tensiones entre la práctica y el discurso del

Tatnall, A., Visscher, A. J., Finegan, A., \& O'Mahony (Ed.), Evolution of Information Technology in Educational Managenent (pp. 107-116). Boston, MA: Springer.

Prestridge, S. (2012). The beliefs behind the teacher that influences their ICT practices. Computers \& Education, 58, 449-458. doi: https://doi.org/10.1016/j.compedu.2011.08.028

Rodrigues, R. (2014). Búsqueda, selección y gestión de información académica de los nativos digitales: Pocas sorpresas y grandes retos educativos. Digital Education Review, (26), 39-60.

Rodríguez Ruiz, Ó. (2005). La triangulación como estrategia de investigación en Ciencias Sociales. Revista de Investigación en Gestión de la Innovación y Tecnología. Recuperado de https://www.madrimasd.org/revista/revista31/tribuna/tribuna2.asp

Sancho Gil, J. M., \& Padilla Petry, P. (2016). Promoting digital competence in secondary education: are schools there? Insights froma a case study. Journal of New Approaches in Educational Research, 6(1), 57-63. doi: https://doi.org/10.7821/naer.2016.1.157

Seguel, C. E. (2015). Evaluación de los estudiantes y docentes sobre las Tecnologías de Información y Comunicación en los procesos de enseñanza-aprendizaje: una mirada desde la carrera de Sociología de la UC Temuco. Un estudio de caso. Digital Education Review, 28, 102-122.

Sevensma, K. (2013). Expanding conceptualizations of online reading comprehension to support at-risk readers. In International Society of Technology in Education's 34th Annual Convention. San Antonio, Texas.

Smith, B. E., Kiili, C., \& Kauppinen, M. (2016). Transmediating argumentation: Students composing across written essays and digital videos in higher education. Computers and Education, 102. https://doi.org/10.1016/j.compedu.2016.08.003

Thereau, J. (2010). Les entretiens d'autoconfrontation et de remise en situation par les traces matérielles et le programme de recherche «cours d'action». Revue d'anthropologie Des Connaissances, 4(2), 287322., 4(2), 287-322.

Tirado-Morueta, R., \& Aguaded-Gómez, J. I. (2014). Influencias de las creencias del profesorado sobre el uso de la tecnología en el aula. Revista de Educacion, (363), 230-255. doi: https://doi.org/10.4438/1988-592X-RE-2012-363-179

Tondeur, J., Van Braak, J., Ertmer, P. A., \& Ottenbreit-Leftwich, A. (2017). Understanding the relationship between teachers' pedagogical beliefs and technology use in education: a systematic review of qualitative evidence. Educational Technology Research and Development, 65(3), 555-575. doi: https://doi.org/10.1007/s11423-016-9481-2

Valverde-Crespo, D., \& González-Sánchez, J. (2016). Búsqueda y selección de información en recursos digitales: Percepciones de alumnos de Física y Química de Educación Secundaria Obligatoria y Bachillerato sobre Wikipedia. Revista Eureka sobre Enseñanza y Divulgación de las Ciencias, 13(1), 67-83.

Watkins, R., Engel, L. C., \& Hastedt, D. (2015). Is the "Net Generation" ready for digital citizenship? Perspectives from the IEA International Computer and Information Literacy Study 2013. Amsterdam: IEA. 